\title{
BMJ Open Frailty in major trauma study (FRAIL-T): a study protocol to determine the feasibility of nurse-led frailty assessment in elderly trauma and the impact on outcome in patients with major trauma
}

\author{
Heather Jarman (D) , ${ }^{1}$ Robert Crouch, ${ }^{2}$ Mark Baxter, ${ }^{3}$ Elaine Cole, ${ }^{4}$ Bebhinn Dillane, \\ Chao Wang ${ }^{5}$
}

To cite: Jarman $\mathrm{H}$, Crouch $\mathrm{R}$, Baxter $\mathrm{M}$, et al. Frailty in major trauma study (FRAIL-T): a study protocol to determine the feasibility of nurse-led frailty assessment in elderly trauma and the impact on outcome in patients with major trauma. BMJ Open 2020;10:e038082. doi:10.1136/ bmjopen-2020-038082

- Prepublication history for this paper is available online. To view these files, please visit the journal online (http://dx.doi org/10.1136/bmjopen-2020 038082).

Received 26 February 2020 Revised 19 May 2020 Accepted 01 June 2020

A Check for updates

(c) Author(s) (or their employer(s)) 2020. Re-use permitted under CC BY-NC. No commercial re-use. See rights and permissions. Published by BMJ.

For numbered affiliations see end of article.

\section{Correspondence to}

Professor Heather Jarman; heather.jarman@stgeorges. nhs.uk

\begin{abstract}
Introduction The burden of frailty on older people is easily recognisable by increasing mortality and morbidity, longer hospital stays and adverse discharge locations. In the UK, frailty screening has recently become part of the best practice commissioning tariff within National Health Service England, yet there is no evidence or consensus as to who should carry out this assessment or within which time frame. As major trauma is an increasing burden for older people, there is a need to focus clinician's attention on early identification of frailty in the emergency department (ED) in patients with major trauma as a way to underpin frailty specific major trauma pathways, to optimise recovery and improve patient experience. Throughout the patient with major trauma pathway, nurses are perhaps best placed to conduct timely clinical assessments working with the patient, family and multidisciplinary team to influence ongoing care. This study aims to determine the feasibility of nurse-led assessment of frailty in patients aged 65 years or more admitted to major trauma centres (MTCs).
\end{abstract}

Methods and analysis This is a prospective observational study conducted across five UK MTCs, enrolling 370 participants over 9 months. The primary aim is to determine the feasibility of nurse-led frailty assessment in MTC EDs in patients aged 65 years or more following traumatic injury. The prevalence of frailty and the best assessment tool for use in the ED will be determined. Other outcome measures include quality of life and frailty assessment 6 months after injury, mortality and discharge outcomes.

Ethics and dissemination The study was given ethical approval by the Social Care Research Ethics Committee (REC no 19/EC08/0006). Findings will be published in scientific journals and presented to national and international conferences.

Trial registration number ISRCTN10671514.

\section{INTRODUCTION}

There is no universal definition of frailty, but it is regarded as a condition characterised by
Strengths and limitations of this study

- Feasibility and acceptability of frailty assessment tools in patients with major trauma will be measured and compared.

- The use of different assessment methods, correlated against an expert assessment from a geriatrician, will enable us to recommend which is the most suitable tool for use in this patient group.

- Recruiting from major trauma centres only limits the generalisability and transferability of the findings.

a cumulative decline of physiological resilience across a number of body systems. ${ }^{1-3}$ A key principle is that people who are frail are at risk of significant functional, physical and cognitive decline following an episode of illness or injury. ${ }^{4}$ The prevalence of frailty is dependent on the definition used and how it is measured. A literature review by Sternberg et a $\tilde{\varphi}$ cites rates of $5 \%-58 \%$, with the prevalence increasing with age. In the UK, Gale $e t$ $a t^{6}$ estimate that in 60-69years old the rate is $6.5 \%$, rising to $65 \%$ in the over $90 \mathrm{~s}$.

In 2016 in the UK, $18 \%$ of people were aged 65 years and over. ${ }^{7}$ As people are living longer and remaining physically fit they follow more active and independent lifestyles resulting in an increased risk of trauma. More elderly people are sustaining major traumatic injury, with the UK incidence in over $75 \mathrm{~s}$ rising from $8.1 \%$ in 1990 to $26.9 \%$ in $2013 .{ }^{89}$ Data from major trauma centres (MTCs) in London for the last 2 years show patients age 65 and over accounted for $31 \%$ (1636 of 5348 patients) of major trauma activity in 2016, rising to $33 \%$ (1788 of 5486 patients) in 2017. There are similar findings for patients aged 65 and over 
in published data worldwide, with falls being the most common cause of injury. These low energy falls, while unremarkable in the younger population, result in disproportionate injuries and mortality rates in the elderly who may have multiple comorbidities and frailty. ${ }^{40}$ International evidence suggests two 'groups' of elderly patients with trauma exist-those functioning well physically prior to the injury, and those who are frail. It is preinjury frailty status, as well as age, that appear to influence outcome with those who are frail suffering worse in-hospital complications and increased mortality. ${ }^{11}{ }^{12}$ Despite the available evidence on the significance of frailty on outcome there is a lack of evidence describing the characteristics and effects of frailty in major trauma in the UK. We currently do not know how many severely injured patients are affected by frailty. This could be due to the lack of consensus in defining frailty and lack of standardisation in how it is measured and recorded. Severely injured older people are initially cared for in MTC emergency departments (EDs), yet a recent international scoping review reported only $14 \%$ of patients were frailty screened during this phase of care. ${ }^{13}$ This may be due to the fact that not all elderly trauma patients 'appear' frail, or that some trauma-specific frailty tools are time and resource intensive to complete, or frailty screening is only recommended as part of a comprehensive geriatric assessment at a later stage in the patients' journey. ${ }^{14}$ However, a recent study has reported significantly improved outcomes in frail older patients with trauma who have early frailty screening and expedited care via a specialist pathway. ${ }^{15}$

There is no clear consensus on the best way to measure frailty. This has led to a lack of consistency in the definitions and measures used to provide outcome data in the various patient groups. The British Geriatric Society ${ }^{1}$ makes recommendations for frailty assessment in different clinical groups but do not recommend a specific tool for use in major trauma.

Major trauma is an increasing burden for older people; therefore, we wish to focus attention on early identification of frailty in the ED in patients with major trauma as a way to underpin frailty specific major trauma pathways, so these may optimise recovery and improve patient experience. We do not currently know the prevalence of frailty in the UK major trauma population or whether it is feasible to carry out accurate frailty assessment in the ED in this patient group. As nurses are involved in every stage of the patients with major trauma pathway we feel they are best placed to provide an early clinical assessment of frailty, working with the patient, family and wider multiprofessional team to influence ongoing care.

The aim of this study is to evaluate the feasibility of nurse-led assessment of frailty in the ED in patients aged 65 years or over admitted to MTCs. We will demonstrate if this assessment is accurate (when compared with geriatrician assessment) and if there is a nurse preference for a particular measure of frailty. This study will also assist in building a picture of the older major trauma population and their outcomes over time.

\section{STUDY OBJECTIVES}

1. Evaluate the feasibility of completing nurse-led assessment of frailty in the ED of patients aged 65 years and over admitted to MTCs following traumatic injury.

2. Explore the relationship between early nurse-led frailty assessment and geriatrician frailty assessment within 72 hours of admission.

3. Determine the prevalence of frailty and characteristics of patients aged 65 years and over admitted to MTCs in the UK.

4. Examine the relationship between early identification of frailty and outcomes in major trauma patients aged 65 years and over.

\section{METHODS AND ANALYSIS}

This is a prospective observational study of major trauma patients aged 65 and over admitted to MTCs.

\section{Setting and participants}

Patients will be recruited from five MTCs (level 1 trauma centre equivalent hospitals) over a 10 -month period from June 2019 to March 2020, with study completion in September 2020 after a 6-month follow-up period. Patients will be eligible to take part if they are aged 65 years or over, are admitted to a MTC and require activation of the hospitals' trauma team. Patients who are transferred from another hospital or who are discharged directly from ED will be excluded. Eligible patients identified by the clinical team working in the ED will be approached to participate in the study. Patients will be assessed for their capacity to consent to the study by their treating clinician prior to enrolment and guidance on consent in emergency settings will be used. ${ }^{16}$ Consent will be received by a member of the research team at each site. This could be participant consent in those with capacity, or personal or professional consultee opinion in those who lack capacity. Study participants will then be assessed for frailty by a nurse who has been trained to use the assessment tools. This will occur in addition to the patient's usual care. Patients will follow the standard admission pathway within each MTC.

\section{Sample size}

Data from London-based geriatricians working with patients with major trauma suggest that frailty affects approximately $37 \%$ of those aged 65 years or over. In the sample size calculation, we use this percentage of frailty as the outcome variable, and to accurately estimate the prevalence it was determined that we need a sample size of 370 to achieve a $95 \%$ CI ( $32 \%$ to $42 \%)$ at $97 \%$ probability of achieving the target precision. 


\section{Outcomes}

The primary outcome is identification of frailty in the ED using three different assessment tools. This will be determined by the percentage of patients where a score can be derived from each tool. As the measures of frailty are all different we will use each tool, if completed, to categorise patients as either 'frail' or 'not frail' according to the cutoff for each tool. Accuracy of the ED assessment will be determined by comparison of nurse assessment of frailty using each tool with that of a senior geriatrician using the Clinical Frailty Scale (CFS) ${ }^{17}$ carried out as part of standard of care. Measuring frailty in the patient group will also allow us to determine the prevalence of frailty.

Secondary outcomes:

Other outcomes that will enable us to determine the characteristics of this patient group are:

- Quality of life at hospital discharge and 6 months postinjury, and patient or carer reported frailty status at 6 months postinjury.

- Rate of in-hospital deaths and those occurring within 6 months from discharge.

- Discharge destination from hospital compared with preadmission.

\section{Data collection}

At recruitment to the study, baseline data will be recorded by nurses trained to use the frailty assessment tools using information reported by the patient or carer, and taken from the patients' clinical records. Training in the use of the individual frailty assessment tools will be provided at each site by the study coordinator. To achieve the aim of assessing the feasibility of carrying out frailty assessments in the ED, frailty assessment tools will be administered prior to the patient leaving the ED using any information that is available (patient, carer, medical records, nurses' clinical judgement). Nurses will be asked to rank the ease of use and preference for each tool for each individual participant to ascertain acceptability of use.

Patients will be followed up in-hospital to death, discharge or 12 weeks following admission. Six-month follow-up data will be obtained by the study coordinator from patients or relatives using the preferred method identified prior to hospital discharge (letter, phone or email). Patients' mortality status at 5 months from injury will be checked using electronic records prior to any attempt to contact the patient or relative. The full list of data collection points and time frames are presented in table 1 .

\section{Assessment tools}

Frailty will be assessed using three standardised measures.

1. Trauma Specific Frailty Index (TSFI): composed of 15 questions designed to assess frailty in the trauma setting. ${ }^{18}$ It can be completed by participants, or next of kin if the participant lacks capacity. A TSFI score of $>0.27$ is found to be an independent predictor of unfavourable outcomes after trauma in this patient cohort.
2. Preferred Reporting Items for Systematic Reviews and Meta-Analyses-7: a self-report questionnaire composed of seven unambiguous questions aimed at identifying frail older adults. It utilises closed questions, 'yes' or 'no' answers, and a score of three or more identifies frailty. ${ }^{19}$

3. CFS: chosen as it forms part of the routine measurement of frailty by geriatricians in the National Health Service (NHS) Best Practice Tariff for Major Trauma from April 2019. It is a validated tool used to assess frailty in the elderly population and is based on clinical judgement. ${ }^{17}$ It uses nine pictorial representations alongside a short descriptor to assign a frailty score: 1 (very fit) to 9 (terminally ill). Participants scoring 5 or more are considered to be frail.

The patient's injury severity will be determined by the patient's a Abbreviated Injury Score (AIS) and Injury Severity Score (ISS) on discharge from hospital or death. AIS classifies the severity of injury in each of six defined body regions (head and neck, face, chest, abdomen, pelvis and extremities). ISS is an anatomical scoring system that standardises the severity of traumatic injury. ${ }^{20}$

To meet the secondary outcome of the study in evaluating health-related quality of life this will be measured using the EuroQoL-5 dimensions-5 levels, known as EQ-5D. ${ }^{21}$ It will be completed with the patient or closest relative at the point of discharge or on the 12th week of admission for long-term hospital stays and repeated at 6 months postinjury.

\section{Data analysis}

Data will be analysed using statistical software. Comparisons of continuous data such as age, dates, times, number of pre-existing medications, Glasgow Coma Scale, systolic blood pressure, temperature, heart rate, lactate, ISS, length of stay and numerical frailty scores will be conducted using unpaired Student's t tests or MannWhitney $\mathrm{U}$ tests (for parametric and non-parametric analysis, respectively). Analysis of more than two groups of continuous data will be conducted using analysis of variance or Kruskal-Wallis tests. Analysis of categorical data such as gender, ethnicity, comorbidities, mechanism and location of injury, usual residence and onwards discharge destination, EQ-5D categories, frailty categories, mortality and nurse rating will be analysed using $\mathrm{X}^{2}$ or Fisher's exact tests. A $\mathrm{p}<0.05$ is considered statistically significant, and its corresponding false discovery rate using the Benjamini-Hochberg method is reported to account for multiplicity. Feasibility will be reported as the proportion of tests completed for each patient within the ED time frame, and by thematic analysis of the qualitative feedback from the nursing staff. The relationship between CFS scores by nurses and geriatricians will be examined using Spearman's correlation coefficient and kappa statistic for measuring the inter-rater agreement. The clinical outcomes will be reported as descriptive statistics (counts and per cent). Descriptive summaries of 
Table 1 Data collected in the Frailty in Major Trauma study (FRAIL-T) study

\begin{tabular}{|c|c|c|c|c|}
\hline & \multirow{2}{*}{$\begin{array}{l}\text { Baseline } \\
\text { While in } \\
\text { ED }\end{array}$} & \multicolumn{3}{|l|}{ Follow-up } \\
\hline & & $\begin{array}{l}\text { With } 72 \text { hours } \\
\text { of admission }\end{array}$ & $\begin{array}{l}\text { On discharge/death } \\
\text { or transfer from } \\
\text { hospital }\end{array}$ & $\begin{array}{l}6 \text { months } \\
\text { postinjury }\end{array}$ \\
\hline Sociodemographic data: age, gender, ethnicity & $x$ & & & \\
\hline Residential status (current type of residence) & $\mathrm{X}$ & & & $x$ \\
\hline $\begin{array}{l}\text { Comorbidities and no of regular significant preinjury } \\
\text { medications }\end{array}$ & $x$ & & & \\
\hline $\begin{array}{l}\text { Injury and clinical characteristics: date and time of ED } \\
\text { attendance, trauma call information, further injury information } \\
\text { including mechanism of injury and location }\end{array}$ & $\mathrm{x}$ & & & \\
\hline Lactate measurement (if taken as part of routine care) & $\mathrm{X}$ & & & \\
\hline $\begin{array}{l}\text { First set of vital signs: Glasgow Coma Scale, systolic blood } \\
\text { pressure, temperature, heart rate }\end{array}$ & $\mathrm{x}$ & & & \\
\hline \multicolumn{5}{|l|}{ Assessment of frailty } \\
\hline e-FI (from primary care record) & $x$ & & & $x$ \\
\hline Trauma Specific Frailty Index & $x$ & & & $x$ \\
\hline PRISMA 7 & $x$ & & & $x$ \\
\hline Clinical Frailty Scale (CFS) & $x$ & & & $x$ \\
\hline Nurse acceptability and rating of frailty tools & $x$ & & & \\
\hline Geriatrician assessment of frailty using CFS & & $x$ & & \\
\hline \multicolumn{5}{|l|}{ Outcome data } \\
\hline $\begin{array}{l}\text { Hospital length of stay: date and location of discharge or } \\
\text { transfer, critical care length of stay-level two and three- } \\
\text { and total length of hospital stay in the MTC }\end{array}$ & & & $x$ & \\
\hline Injury Severity Score & & & $\mathrm{X}$ & \\
\hline Health-related quality of life assessment & & & $x$ & $x$ \\
\hline Hospital readmissions & & & & $x$ \\
\hline Date and cause of death (if applicable) & & & & $\mathrm{x}$ \\
\hline
\end{tabular}

ED, emergency department; e-FI, electronic Frailty Index; FRAILT, The Frailty in Major Trauma Study; MTC, Major Trauma Centres; PRISMA, Preferred Reporting Items for Systematic Reviews and Meta-Analyses.

the changes to frailty scores and quality of life over time will be presented.

\section{PATIENT AND PUBLIC INVOLVEMENT}

Members of a patient and public research expert group have been involved in the design of the study. They provided advice on the use of personal consultees for patients lacking capacity and on the content of the patient information material. They also provided comments on acceptability of the frailty assessment tools used in the study from the perspective of the patients and carers, both the time taken to complete and the questions within each tool.

\section{ETHICS AND DISSEMINATION}

The study obtained an ethical opinion for conduct by the Social Care Research Ethics Committee (REC no 19/IEC08/0006). Consent guidance for undertaking research in emergency settings and with patients lacking capacity will be followed.

For patients with capacity informed consent will be obtained as a two-part process prior to inclusion into the study. As patients presenting to MTCs may not be able to read and complete a written consent process within the time they are in the ED, verbal consent will be obtained for the frailty measurement tools to be completed. Patients will subsequently be given a written information sheet and the study team will be available to answer any questions. If they still wish to take part they will then go through the written consent process. This will be deferred written consent for frailty assessment and written consent for the other aspects of the study. If they choose to end their participation at this point then any data already collected will be destroyed.

Some participants eligible for the study may have impaired capacity at the time of attendance. If a personal consultee is available then personal consultee advice will 
be sought. In line with UK guidance on consent in emergency situations, a nominated consultee will be used if a personal consultee is not available. ${ }^{16}$ If the nominated consultee is unable to provide advice on the patient's participation, the patient will not be enrolled in the study and no information will be collected. If and when clinically appropriate, consent will be sought from the patient.

All patient information will be identified using only a study ID number. Procedures for handling data will be in accordance with the UK Data Protection Act 2018. Data will be collected on a paper CRF and entered as soon as possible into a password-protected study database (Research Electronic Data Capture - REDCap) by the local study team. A password-protected coding log will be used which will include identifiable participant data (name, address and date of birth) for patients who have consented to long-term outcome follow-up. This will be transferred to the study coordinator at the end of the in-hospital data collection period. At 5 months after injury the NHS Health and Social Care Information Centre (NHS Spine) or participants general practitioner will be contacted to ensure that the participant is still alive at this time point. This will avoid erroneous long-term follow-up and exposing relatives to undue distress.

As this is an observational study there will be no anticipated safety events as a result of patient participation. Notification of death is not recorded as an adverse event, it is reported as an outcome if occurs. Patient-specific concerns will be raised with the principal investigator at each site and escalated to the chief investigator as required.

The study management committee will meet regularly and is responsible for monitoring all aspects of the conduct and progress of the study, ensuring the protocol is adhered to and taking appropriate action to safeguard participants and the quality of the study itself. Amendments of any changes to the study will be submitted to the relevant ethics committee.

Findings from the study will be published in peerreviewed journals and presented nationally and internationally. We will disseminate findings through appropriate networks and specialist groups.

\section{Author affiliations \\ ${ }^{1}$ Emergency Department Clinical Research Unit, St George's University Hospitals NHS Foundation Trust, London, UK \\ ${ }^{2}$ Emergency Department, University Hospital Southampton NHS Foundation Trust, Southampton, UK \\ ${ }^{3}$ Geriatric Medicine, University Hospital Southampton NHS Foundation Trust, Southampton, UK \\ ${ }^{4}$ Blizard Institute, Queen Mary University of London, London, UK \\ ${ }^{5}$ Kingston University Faculty of Health Social Care and Education, London, UK}

Twitter Heather Jarman @godalmingjarman, Bebhinn Dillane @bbebhinn and Chao Wang @excel_wang

Acknowledgements We would like to thank members of the Centre for Public Engagement, part of the Faculty of Health, Social Care and Education, Kingston University and St George's, University of London, London, UK, for their contribution to the development of the protocol and data collection tools. The following members of the Pan-London Elderly Major Trauma Group provided advice and consensus opinions on the use of frailty tools and the recruitment processes: Trish Burton, Cara Jennings, George Peck, Dhanupriya Sivapathasuntharam, Rhonda Sturley, Jane Tippett.

Contributors $\mathrm{HJ}, \mathrm{RC}, \mathrm{MB}$ and $\mathrm{EC}$ contributed to the conception and design of the study. $\mathrm{HJ}$ and $\mathrm{BD}$ drafted the manuscript. $\mathrm{BD}$ is managing day to day research activities. CW completed the data analysis section of the manuscript and will EC developed the analysis plan. $\mathrm{HJ}, \mathrm{RC}, \mathrm{MB}, \mathrm{EC}, \mathrm{BD}$ and $\mathrm{CW}$ read and approved the final manuscript.

Funding This work was supported by The Burdett Trust for Nursing. The study is sponsored by St George's University Hospitals NHS Foundation Trust.

Competing interests None declared.

Patient and public involvement Patients and/or the public were involved in the design, or conduct, or reporting, or dissemination plans of this research. Refer to the Methods section for further details.

Patient consent for publication Not required.

Provenance and peer review Not commissioned; externally peer reviewed.

Open access This is an open access article distributed in accordance with the Creative Commons Attribution Non Commercial (CC BY-NC 4.0) license, which permits others to distribute, remix, adapt, build upon this work non-commercially, and license their derivative works on different terms, provided the original work is properly cited, appropriate credit is given, any changes made indicated, and the use is non-commercial. See: http://creativecommons.org/licenses/by-nc/4.0/.

ORCID iD

Heather Jarman http://orcid.org/0000-0002-4820-3291

\section{REFERENCES}

1 British Geriatric Society. Fit for Frailty - consensus best practice guidance for the care of older people living in community and outpatient settings - a report from the British Geriatrics Society, 2014. Available: http://www.bgs.org.uk/campaigns/fff/fff_full.pdf

2 Dent E, Kowal P, Hoogendijk EO. Frailty measurement in research and clinical practice: a review. Eur J Intern Med 2016;31:3-10.

3 Junius-Walker $U$, Onder G, Soleymani D, et al. The essence of frailty: a systematic review and qualitative synthesis on frailty concepts and definitions. Eur J Intern Med 2018;56:3-10.

4 Clegg A, Young J, lliffe S, et al. Frailty in elderly people. Lancet 2013;381:752-62.

5 Sternberg SA, Wershof Schwartz A, Karunananthan S, et al. The identification of frailty: a systematic literature review. J Am Geriatr Soc 2011;59:2129-38.

6 Gale CR, Cooper C, Sayer AA. Prevalence of frailty and disability: findings from the English longitudinal study of ageing. Age Ageing $2015 ; 44: 162-5$.

7 Office of National Statistics. Overview of the UK population, 2017. Available: https://www.ons.gov.uk/peoplepopulationandcommunity/ populationandmigration/populationestimates/articles/overviewofth eukpopulation/july2017

8 Kehoe A, Smith JE, Edwards A, et al. The changing face of major trauma in the UK. Emerg Med J 2015;32:911-5.

9 TARN. Major trauma in older people, 2017. Available: https://www. tarn.ac.uk/content/downloads/3793/Major\%20Trauma\%20in\% 20Older\%20People\%202017.pdf

10 Sterling DA, O'Connor JA, Bonadies J. Geriatric falls: injury severity is high and disproportionate to mechanism. J Trauma 2001;50:116-9.

11 Maxwell CA, Patel MB, Suarez-Rodriguez LC, et al. Frailty and prognostication in geriatric surgery and trauma. Clin Geriatr Med 2019;35:13-26.

12 Vu CCL, Runner RP, Reisman WM, et al. The frail fail: increased mortality and post-operative complications in orthopaedic trauma patients. Injury 2017;48:2443-50.

13 Theou O, Squires E, Mallery K, et al. What do we know about frailty in the acute care setting? A scoping review. BMC Geriatr 2018;18:139.

14 Ellis G, Gardner M, Tsiachristas A, et al. Comprehensive geriatric assessment for older adults admitted to hospital. Cochrane Database Syst Rev 2017;9:CD006211.

15 Engelhardt KE, Reuter Q, Liu J, et al. Frailty screening and a frailty pathway decrease length of stay, loss of independence, and 30 -day readmission rates in frail geriatric trauma and 
emergency general surgery patients. $J$ Trauma Acute Care Surg 2018;85:167-73.

16 DH Scientific Development and Bioethics Division. Guidance on nominating a consultee for research involving adults who lack capacity to consent, 2008.

17 Rockwood K, Song X, Macknight C, et al. A global clinical measure of fitness and frailty in elderly people. CMAJ 2005;173:489-95.

18 Joseph B, Pandit V, Zangbar B, et al. Validating trauma-specific frailty index for geriatric trauma patients: a prospective analysis. J Am Coll Surg 2014;219:10-17.
19 Raîche M, Hébert R, Dubois M-F. PRISMA-7: a case-finding tool to identify older adults with moderate to severe disabilities. Arch Gerontol Geriatr 2008;47:9-18.

20 Baker SP, O'Neill B, Haddon W, et al. The injury severity score: a method for describing patients with multiple injuries and evaluating emergency care. J Trauma 1974;14:187-96.

21 Herdman M, Gudex C, Lloyd A, et al. Development and preliminary testing of the new five-level version of EQ-5D (EQ-5D-5L). Qual Life Res 2011;20:1727-36. 
Correction: Frailty in major trauma study (FRAIL-T): a study

protocol to determine the feasibility of nurse-led frailty

assessment in elderly trauma and the impact on outcome in

patients with major trauma

Jarman H, Crouch R, Baxter M, et al. Frailty in major trauma study (FRAIL-T): a study protocol to determine the feasibility of nurse-led frailty assessment in elderly trauma and the impact on outcome in patients with major trauma. BMJ Open 2020;10:e038082. doi: 10.1136/bmjopen-2020-038082

This article was previously published with error. The authors noticed below error:

On page 3, top of column 2, Reporting Items for Systematic Reviews and MetaAnalyses-7 should read: PRISMA7 (Program of Research to Integrate Services of Autonomy).

Open access This is an open access article distributed in accordance with the Creative Commons Attribution Non Commercial (CC BY-NC 4.0) license, which permits others to distribute, remix, adapt, build upon this work non-commercially, and license their derivative works on different terms, provided the original work is properly cited, appropriate credit is given, any changes made indicated, and the use is non-commercial. See: http://creativecommons.org/licenses/by-nc/4.0/.

(c) Author(s) (or their employer(s)) 2020. Re-use permitted under CC BY-NC. No commercial re-use. See rights and permissions. Published by BMJ.

BMJ Open 2020;10:e038082corr1. doi:10.1136/bmjopen-2020-038082corr1

Check for updates 